\title{
Energy-filtering TEM at high magnification: spatial resolution and detection limits
}

\author{
Werner Grogger ${ }^{\mathrm{a}, *}$, Bernhard Schaffer ${ }^{\mathrm{a}}$, Kannan M. Krishnan ${ }^{\mathrm{b}}$, Ferdinand Hofer $^{\mathrm{a}}$ \\ ${ }^{a}$ Research Institute for Electron Microscopy, Graz University of Technology, Steyrergasse 17, Graz A-8010, Austria \\ ${ }^{\mathrm{b}}$ Lawrence Berkeley National Lab., NCEM, Materials Sciences Div., Berkeley, CA 94720, USA
}

Received 15 August 2002; accepted 3 November 2002

\begin{abstract}
Energy-filtering TEM (EFTEM) has turned out to be a very efficient and rapid tool for the chemical characterization of a specimen on a nanometer and even subnanometer length scale. Especially, the detection and measurement of very thin layers has become a great application of this technique in many materials science fields, e.g. semiconductors and hard disk technology. There, the reliability of compositional profiles is an important issue. However, the experimentally obtainable spatial resolution strongly influences the appearance of a thin layer in an EFTEM image, when dimensions reach subnanometer levels, which mainly leads to a broadening of the layer in the image. This fact has to be taken into account, when measuring the thickness of such a thin layer. Additionally, the convolution decreases contrast which makes the layer less visible in the image and finally determines the detection limit.

In this work we present a systematic study on specifically designed $\mathrm{Mn} / \mathrm{PdMn}$ multilayer test specimens to explore the practical aspects of spatial resolution and detection limits in EFTEM. Although specific to the ionization edges used, we will present general conclusions about the practical limitations in terms of EFTEM spatial resolution. Additionally, work will be shown about low energy-loss imaging of thin oxide layers, where delocalization is the main factor responsible for broadening.
\end{abstract}

(C) 2003 Elsevier Science B.V. All rights reserved.

Keywords: Energy-filtering TEM; Spatial resolution; Detection limit

\section{Introduction}

The applications of transmission electron microscopy (TEM) are more and more focusing on investigations on a nanometer length scale and beyond. Besides structural information from high

\footnotetext{
*Corresponding author. Tel.: +43-316-873-8323; fax: +43316-811596.

E-mail address: werner.grogger@felmi-zfe.at (W. Grogger).
}

resolution TEM (HRTEM), analytical information at these dimensions is in many cases paramount to the understanding of macroscopic functional properties of many modern materials science specimens.

In semiconductor industry miniaturization decreases layer thicknesses down to just a few nanometers, for instance for the gate oxide layers. The quality of these layers, like thickness and homogeneity, is directly related to device 
performance, and therefore needs to be determined accurately. In many fields small particles with thin surface layers play an important role (e.g. catalysts), where the analytical characterization of these layers and their dimensions is of great importance. Fine-grained materials exhibit grain sizes down to some ten nanometers, and are in many cases synthesized by adding small amounts of dopants. These dopants were shown to form monolayer thin phases at the grain boundaries. Furthermore, small inclusions and precipitates in nanocomposites determine the macroscopic behavior of these materials, which also need a careful, accurate, and reliable characterization. In all of these applications it is important to determine the morphology as well as the chemistry of the materials investigated.

During the last decade, energy-filtering TEM (EFTEM) has proven to be a powerful analytical method with its resolution reaching atomic levels. Using this technique, chemical information can rather easily be obtained for all different kinds of materials. From such elemental distribution images critical dimensions of the chemical phases involved can be determined. However, measuring small dimensions accurately from EFTEM images requires some caution, as the obtainable EFTEM resolution tends to broaden features to a certain extent and also strongly influences the detection limits. This paper describes how the terms "spatial resolution" and "detection limit" can be understood in relation to EFTEM imaging, and shows how these terms relate to each other. Two application examples demonstrate the capabilities of EFTEM with regard to resolution and detection limit.

\section{Theoretical considerations}

In EFTEM, post-column energy filters in combination with high resolution TEMs can provide the capability to detect small features, like thin layers or small particles, down to subnanometer dimensions [1-10]. (Other groups also tried to reach nm-resolution but either the results were contradictory or not quite unequivocal [11-13].) Often this detection capability is used to describe the spatial resolution of the technique. When talking about spatial resolution in electron microscopy, in most cases "minimum resolvable distance" is meant. Therefore, it should be recalled, that the term "spatial resolution" is mostly understood as the minimum distance between two objects at which they can still be separated. For the definition of this term, Rayleigh's criterion may be used. There, the minimum resolvable distance is defined as the distance between two objects at which the first diffraction minimum of one overlaps with the first maximum of the other. This definition is equivalent to a contrast drop between the two objects to $73.6 \%$ of the maximum. Contrarily, a term like "detection capability" should be used for the ability to detect small objects.

Far more than in high-resolution TEM, the attainable spatial resolution in EFTEM is influenced by the experimental setup: The energy and the shape of the ionization edge, as well as experimental parameters like the collection angle, influence the obtainable resolution together with instrumental parameters (aberration coefficients of the TEM objective lens) $[6,14,15]$. For instance, delocalization is one of the main factors influencing spatial resolution at low energy-losses.

Additionally to these theoretical limitations, however, there are practical aspects, that also limit the resolution in EFTEM: maximum electron dose, exposure time, specimen drift and instrumental instabilities. When considering, that at high magnifications only a small number of atoms (typically 10-1000) contribute to the signal in a pixel of the final image, it is obvious that accurate instrumental alignments and a careful experimental setup are paramount for good quality results. It should also be noted, that a certain amount of elastic contrast is always transferred to energyfiltered images [15], which can lead to artifacts in elemental distribution images. According to the formulae given in the literature e.g. [6,14] the obtainable spatial resolution in an EFTEM image can be computed. Typical values range from a couple of tenths of a nanometer to a few nanometers.

The dimension of a small object in an EFTEM image is therefore a convolution of the real size 
and the spatial resolution. To illustrate this fact, a rectangular profile (real size) was convoluted with a Gaussian (spatial resolution) and the resulting FWHM was measured (Fig. 1). The key point here is that a certain measured dimension is not directly and unambiguously related to the real dimension of the object. Consequently, the spatial resolution must be known in order to perform reliable dimension measurements around and below $1 \mathrm{~nm}$.

On the other hand, the detection limit in EFTEM is strongly related to and dependent of spatial resolution. The definition of this term is mostly equivalent to the question, whether the peak in the signal arising from a small feature is distinguishable from noise or not. Here too, different criteria may be applied to determine the significance of this peak. The effect of spatial resolution is to broaden the peaks and at the same time to spread their intensity (integrated area under the peak) over a larger range. Shrinking dimensions of a feature (diameter or layer thickness) furthermore lead to a decrease in peak height corresponding to a decrease in the detection limit. The data points in Fig. 2 are again the result of a convolution (see above) and show that having a better spatial resolution allows for the detection of smaller objects and vice versa. Therefore, a value for the spatial resolution must be known in order to estimate detection limits. It should also be noted that spatial resolution can be treated from a pure statistical point of view as it was done in [16].

\section{Experimental}

For experimentally measuring the spatial resolution of EFTEM images a multilayer test specimen was designed and fabricated by UHV ion beam deposition. This specimen consisted of two different layer types $(\mathrm{Mn} / \mathrm{PdMn})$, with the thickness of one $(\mathrm{PdMn})$ kept constant $(3.7 \mathrm{~nm})$, whilst the other one steadily decreased down to about $0.5 \mathrm{~nm}(2.6,2.2,1.8,1.4,1.0,0.47 \mathrm{~nm})$. The layer thicknesses were measured and recalibrated from the deposition parameters by HRTEM. The design of this test specimen makes it possible to determine the detection limit and, in combination with simulations, also yields values for the actual spatial resolution. By changing some acquisition parameters (like collection angle and energy slit width), resolution as well as detection capability can be systematically measured for the two

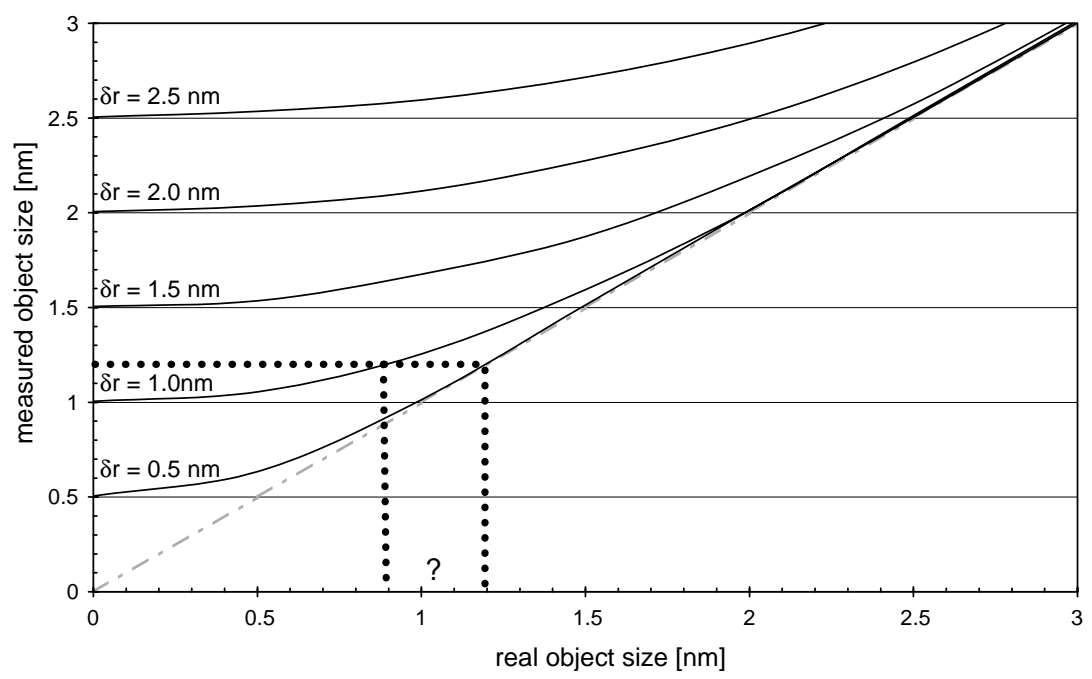

Fig. 1. Apparent size of an object depending on its real size as a function of spatial resolution $(\delta r)$ calculated for rectangular intensity profiles. A certain measured size value for an object (e.g. $1.2 \mathrm{~nm}$ ) is not unambiguously related to its real size (can be anything smaller than $1.2 \mathrm{~nm}$ ). The curve's data points were determined by convoluting a rectangular profile by a Gaussian of width $\delta r$ and measuring the FWHM of the result. 


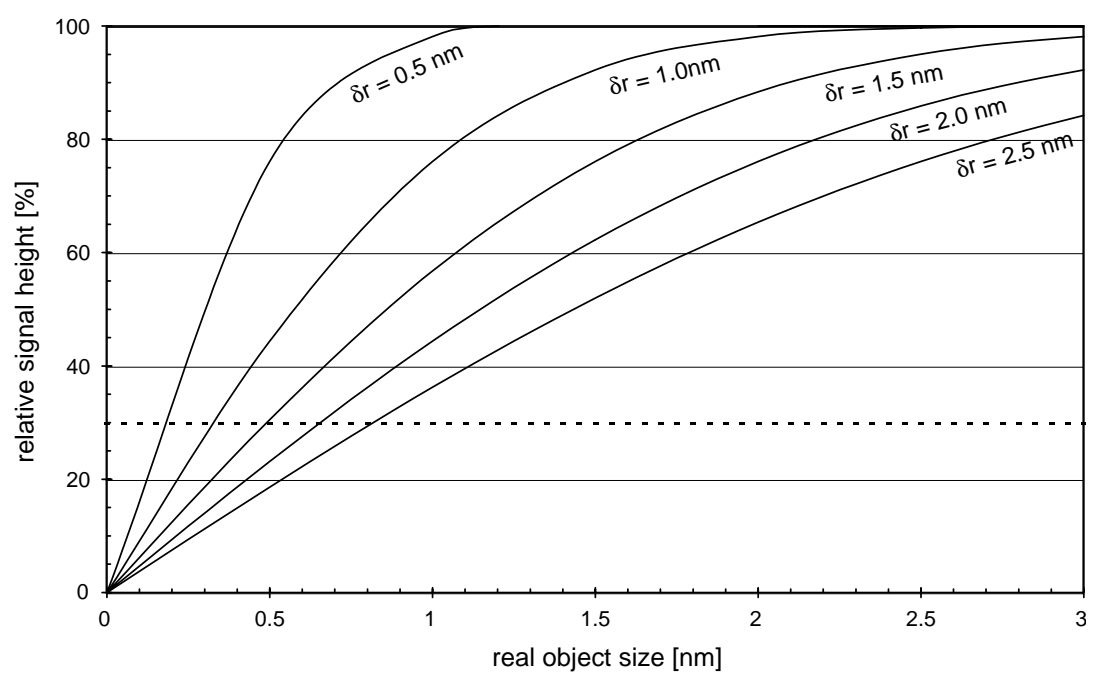

Fig. 2. Relative signal strength from a small object (relative to the signal from a big object) depending on its size as a function of spatial resolution $(\delta r)$ calculated for rectangular intensity profiles and constant chemical composition. Whether or not a small object can be significantly detected strongly depends on spatial resolution. A threshold value for minimum detection may be at $30 \%$, which would allow for an $0.15 \mathrm{~nm}$ small object to be detected when spatial resolution is very good $(0.5 \mathrm{~nm})$. However, a spatial resolution value of $2.5 \mathrm{~nm}$ would decrease this minimum size to around $0.85 \mathrm{~nm}$.

different types of ionization edges of the two elements $\left(\mathrm{Mn}-\mathrm{L}_{23}\right.$ and $\left.\mathrm{Pd}-\mathrm{M}_{45}\right)$. Additionally, an estimate can be given for the detection sensitivity (minimum detectable atomic fraction of an element).

As a second test specimen we used a specially fabricated semiconductor specimen consisting of $\mathrm{SiO}_{2}$ layers of different thicknesses $(1.5,4.5,6.5$, and $12 \mathrm{~nm}$, nominally) with about $50 \mathrm{~nm}$ poly-Si in between. HRTEM was used to measure these thicknesses experimentally. This specimen can be used to test the ability of EFTEM to image the $\mathrm{SiO}_{2}$ layers. Especially, the spatial resolution of single EFTEM images in the low energy-loss regime can be estimated.

Two TEMs were used for this work: A Philips CM200/FEG (Supertwin) at NCEM/LBNL (Berkeley) operated at $200 \mathrm{kV}$ was equipped with a Gatan imaging filter (GIF) using a $1024 \times 1024$ MSC. Additionally, a Tecnai F20 (Supertwin) at FELMI/ZfE (Graz) operated at $200 \mathrm{kV}$ was also used. This microscope is equipped with a high resolution GIF and a $1024 \times 1024$ MSC. All image and data processing was done with Gatan's DigitalMicrograph software. The collection angles were 8.29 and $5.84 \mathrm{mrad}$ for the CM200 and the Tecnai, respectively.

\section{Results}

\subsection{Mn/PdMn multilayer specimen}

In Fig. 3 EFTEM images from the $\mathrm{Mn} / \mathrm{PdMn}$ multilayer specimen are shown: elemental maps (three window technique) as well as jump ratio images (two window technique) [17,18]. The acquisition parameters for these images are listed in (Table 1).

In the elemental maps and jump ratio images $(b$, c, e, f) all layers can be clearly seen down to the thinnest Mn layer. The average specimen thickness can be estimated from the relative thickness map (Fig. 3d) and is around 0.7 (corresponding to approximately $80 \mathrm{~nm}$ ). Drawing a lineprofile on a selection of the images from Fig. 3 and integrating it over 100 pixels reveals the corresponding peaks for all layers (for Mn see Fig. 4, for Pd Fig. 5). The images (and the lineprofiles) of the elemental maps 

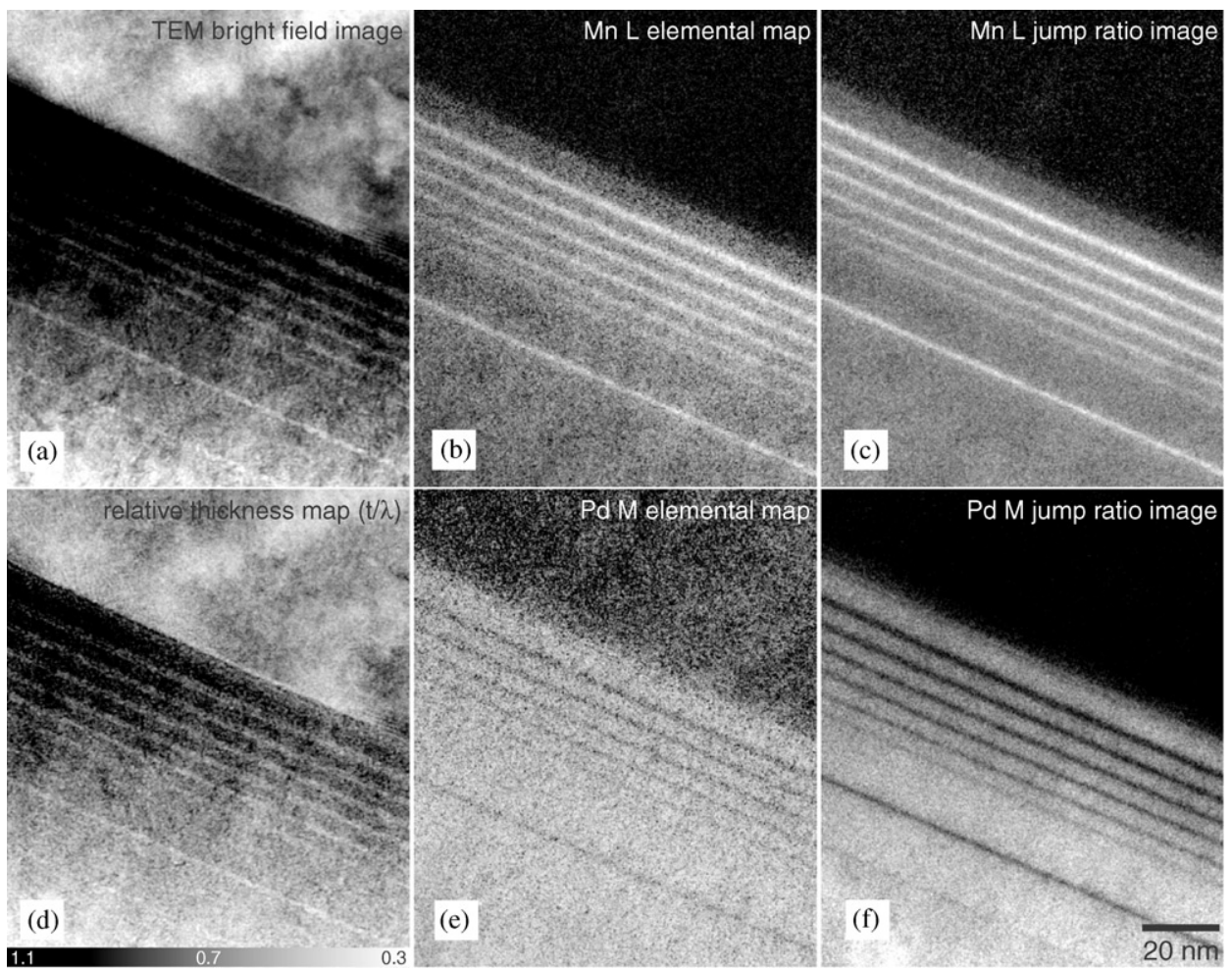

Fig. 3. EFTEM images of a Mn/PdMn specimen: (a) bright field image, (b) Mn elemental map (Mn- $\mathrm{L}_{23}$ ), (c) Mn jump ratio image $\left(\mathrm{Mn}-\mathrm{L}_{23}\right)$, (d) relative thickness $(t / \lambda)$ map, (e) Pd elemental map $\left(\mathrm{Pd}-\mathrm{M}_{45}\right)$, (f) Pd jump ratio image (Pd-M $\left.\mathrm{M}_{45}\right)$. The $\mathrm{Mn}$ layer thicknesses are: 2.6, 2.2, 1.8, 1.4, 1.0, and $0.47 \mathrm{~nm}$. Even the thinnest Mn layer can be seen clearly in (b), (c), (e), and (f).

Table 1

Acquisition parameters for the EFTEM images of Mn and Pd (Fig. 3).

\begin{tabular}{lllllll}
\hline Element & Ion. edge & Pre-edge 1 $(\mathrm{eV})$ & Pre-edge 2 $(\mathrm{eV})$ & Post-edge $(\mathrm{eV})$ & Slit width $(\mathrm{eV})$ & Exposure $(\mathrm{s})$ \\
\hline $\mathrm{Mn}$ & $\mathrm{L}_{23}$ & 607 & 627 & 650 & 20 & 10 \\
$\mathrm{Pd}$ & $\mathrm{M}_{45}$ & 302 & 322 & 408 & 20 & 10 \\
\hline
\end{tabular}

are a little bit more noisy than the jump ratio images.

Also given in these figures are ratio images calculated from the two images that were acquired in front of the ionization edges for background extrapolation (pre-edge images). Such ratio images give a good indication, whether the contrast in an elemental map or jump ratio image is really related to elemental variations. The lineprofiles from these ratio images are showing just noise, so the contrast seen in the elemental jump ratio images is elemental contrast only.
The integrated profiles across the images show peaks for every single layer. As the layers get thinner, the widths of the peaks remain constant for layer thicknesses below about $1.5 \mathrm{~nm}$ (Fig. 6). Therefore, the spatial resolution can be estimated to be about $1.5 \mathrm{~nm}$ and in this case is mainly determined by spherical and chromatic aberration. For thinner layers the area under the peak decreases linearly with layer thickness, until it may reach a level, where it cannot be longer distinguished from noise (e.g. Rose criterion). For the significance of the peak another statistical 

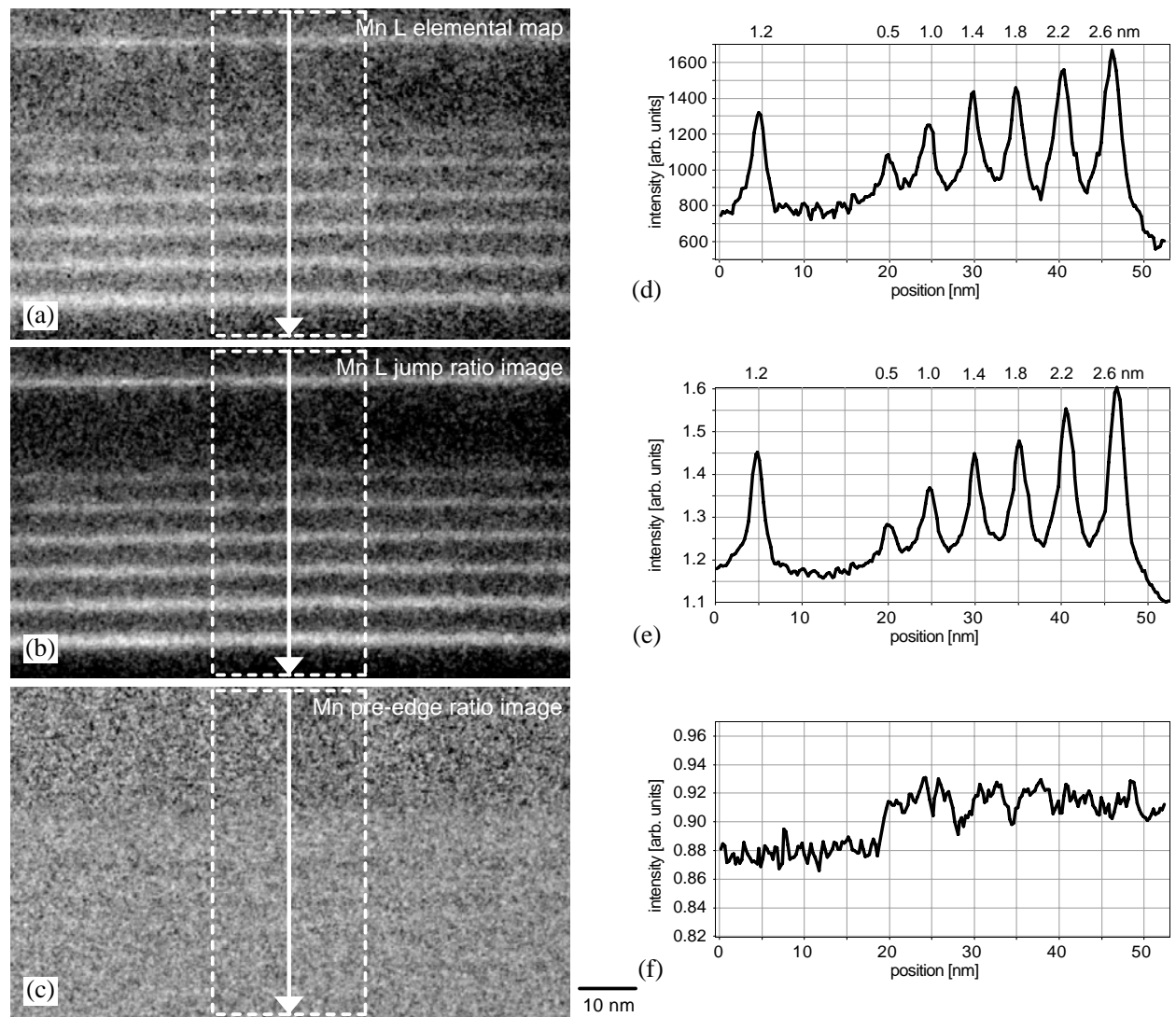

Fig. 4. Mn elemental distribution maps of a Mn/PdMn multilayer and integrated lineprofiles (100 pixels): (a) Mn elemental map (Mn- $\left.\mathrm{L}_{23}\right)$, (b) Mn jump ratio image $\left(\mathrm{Mn}-\mathrm{L}_{23}\right)$, (c) jump ratio image of the pre-edge images (in front of the Mn- $\mathrm{L}_{23}$ edge), (d) lineprofile of (a), (e) lineprofile of (b), lineprofile of (c).

criterion can be used, where the peak intensity must be greater than three times the standard deviation of the background under the peak [19]. Using this criterion a minimum detectable $\mathrm{Mn}$ layer thickness of approximately $0.1 \mathrm{~nm}$ can be estimated from the Mn elemental map (for the acquisition and integration parameters used and for a 100 pixel integrated lineprofile).

\subsection{Semiconductor specimen}

For many applications it is important to characterize thin oxide layers, e.g. in semiconductor devices, which nowadays reach thicknesses of just a few monolayers. The thickness of such layers in semiconductor devices plays an essential role in the functionality of the device.
Using energy-losses in the low loss region (below $100 \mathrm{eV}$ ) for EFTEM imaging we carried out a systematic study on thin $\mathrm{SiO}_{2}$ layers on a Silicon wafer and in between polycrystalline $\mathrm{Si}$ layers [20]. Using the contrast difference between the Si substrate and the thin silicon oxide layer at certain energy-losses, the oxide layers can be imaged (Fig. 7). The main contrast mechanism is believed to be due to the difference in the plasmon region. At these low energy-losses the signal-tonoise ratio is very high, which allows short acquisition times.

However, delocalization in this energy regime is said to deteriorate the resolution to values of a few $\mathrm{nm}[6,14]$. The thickness of the thinnest $\mathrm{SiO}_{2}$ layer was estimated from HRTEM images to be $1.4 \mathrm{~nm}$. The measured values for the FWHM of this peak 

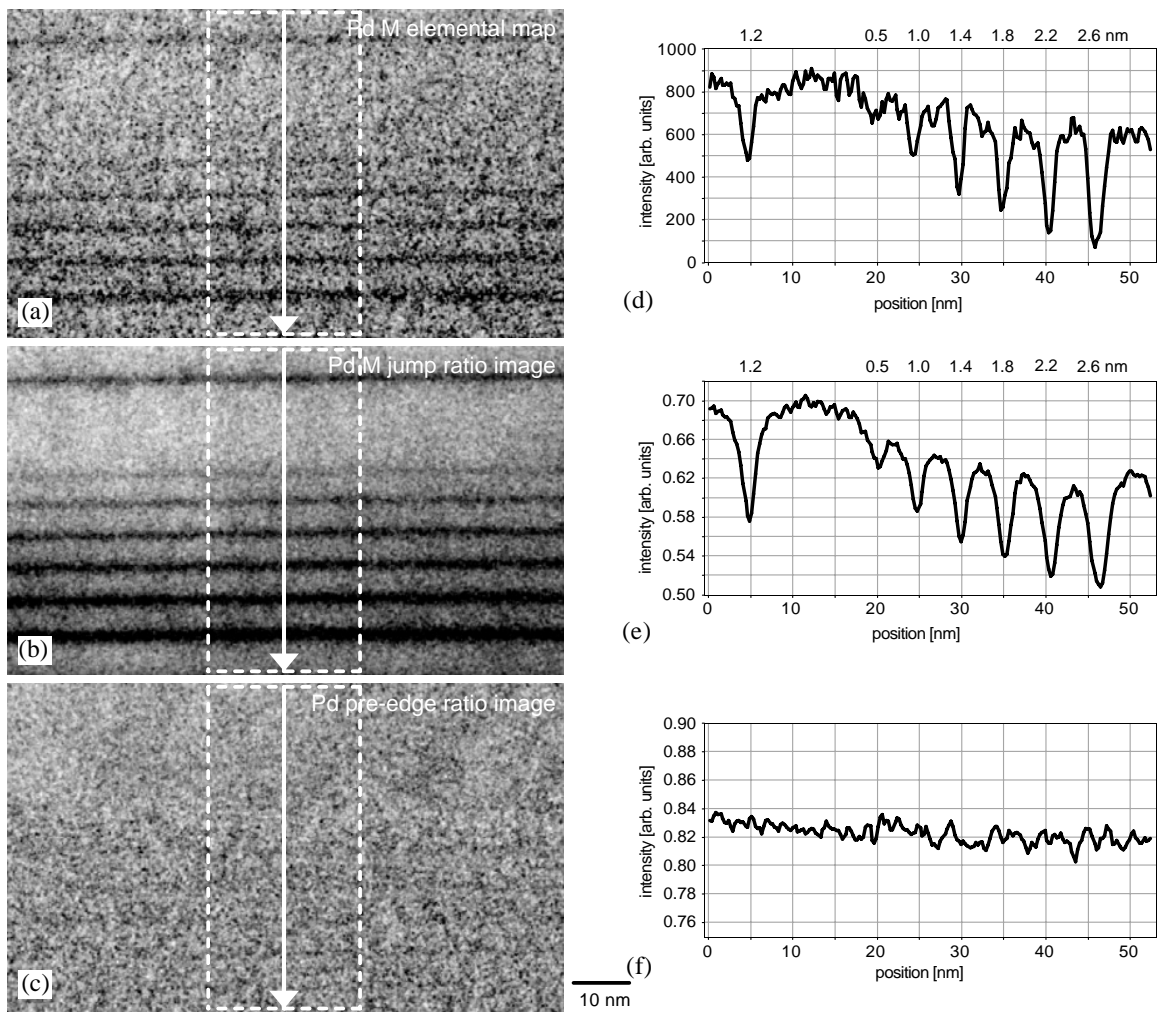

(d)

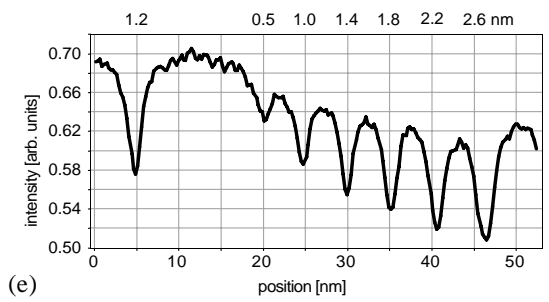

(e)

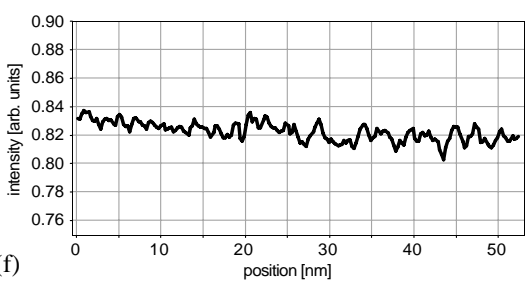

Fig. 5. Pd elemental distribution maps of $\mathrm{Mn} / \mathrm{PdMn}$ multilayer and integrated lineprofiles (100 pixels): (a) Pd elemental map (Pd-M 45 ), (b) Pd jump ratio image $\left(\mathrm{Pd}-\mathrm{M}_{45}\right)$, (c) jump ratio image of the pre-edge images (in front of the Pd-M $\mathrm{M}_{45}$ edge), (d) lineprofile of (a), (e) lineprofile of (b), lineprofile of (c).

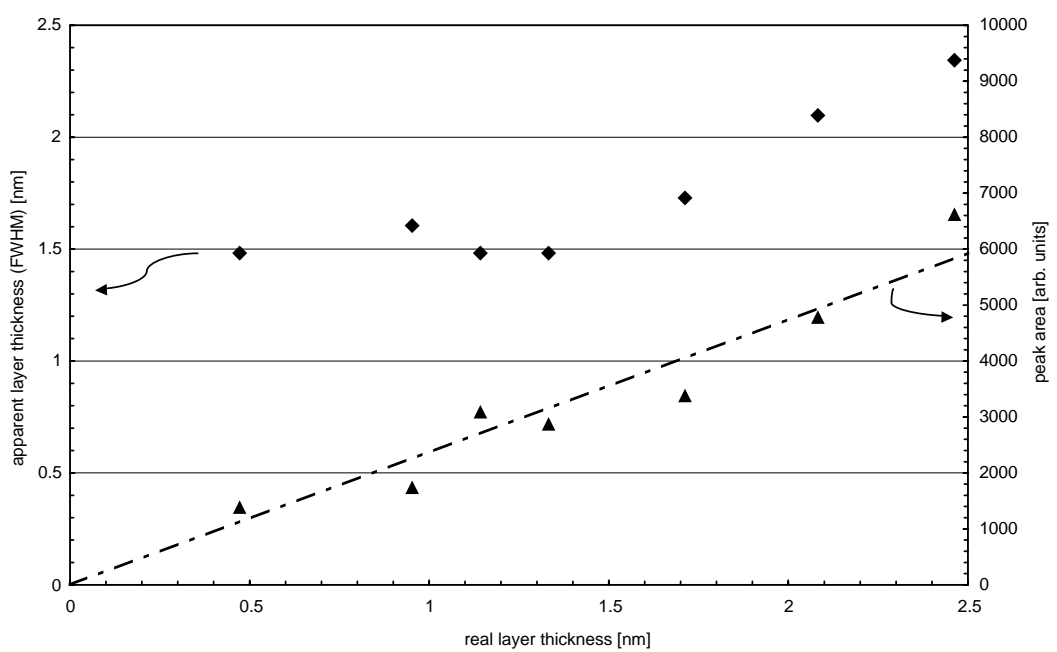

Fig. 6. Evaluation of the Mn elemental map. In this chart the measured width of the peaks (FWHM) and the area under the peaks are plotted versus the "real" layer thicknesses. 

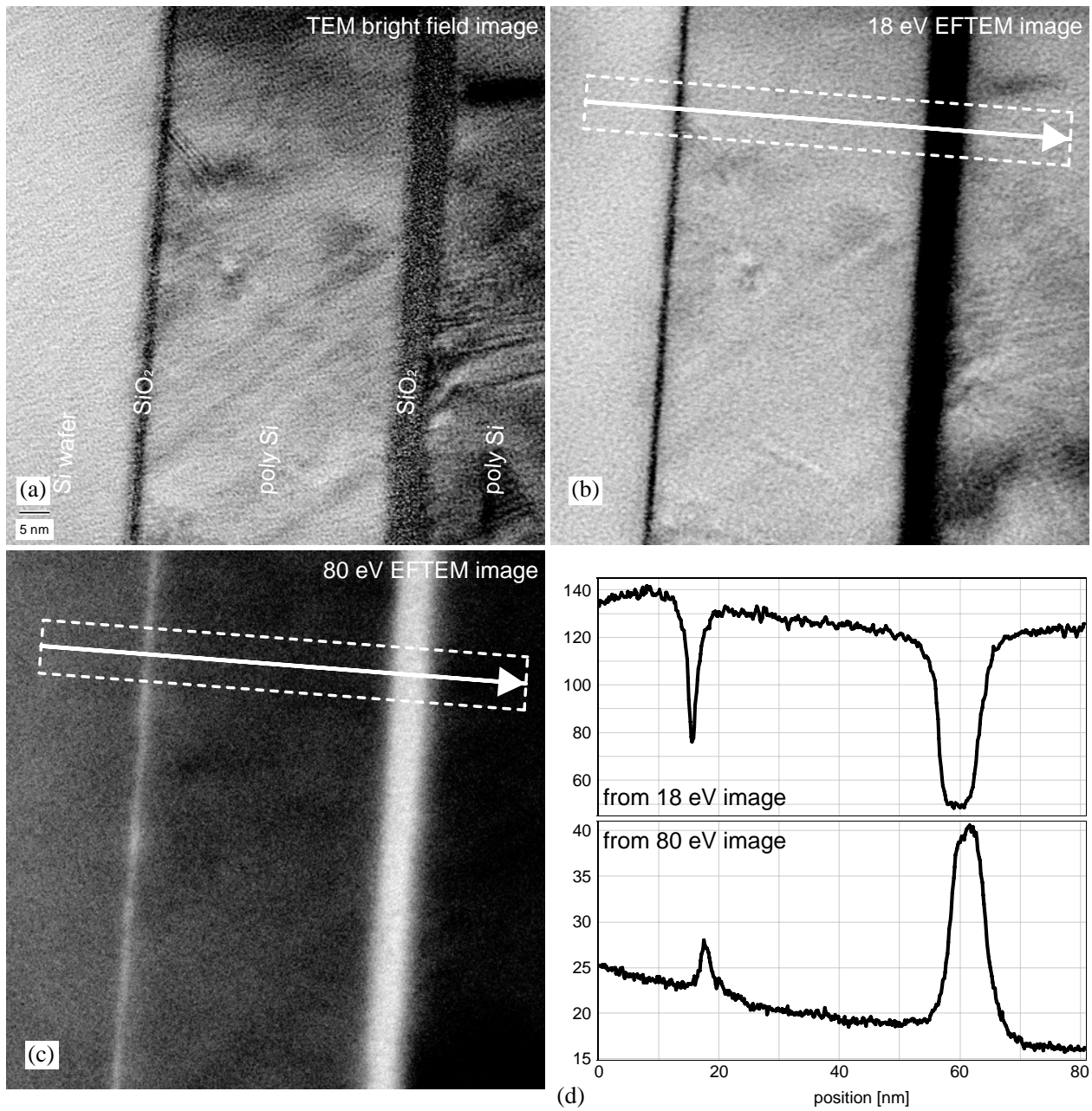

Fig. 7. EFTEM images of $\mathrm{SiO}_{2}$ layers in between $\mathrm{Si}$ (a) bright field image, (b) energy-filtered image at $18 \mathrm{eV}$ (slit size: $5 \mathrm{eV}$ ), (c) energyfiltered image at $80 \mathrm{eV}$ (slit size: $20 \mathrm{eV}$ ), and (d) integrated lineprofiles (100 pixels) drawn across images (b) and (c).

shown in Fig. 7d are almost identical for both energy-losses and were determined to be $1.6 \mathrm{~nm}$. Using the formulae given in the literature the effect of delocalization can be calculated and the resulting spatial resolution can be estimated to be $4.7 \mathrm{~nm}$ (FWHM value, [6]) and $2.1 \mathrm{~nm}\left(\mathrm{~d}_{50 \%}\right.$ value, [14]), respectively (Ref. [6] uses a formula from [21], which was later replaced by an objectfunction description [22], which yielded much smaller delocalization values around and below $0.1 \mathrm{~nm})$. On the other hand, there are other reports [23] stating that the effect of delocalization is in many cases overestimated and the practical consequences are much less than expected as most of the intensity is located in the extended tails of the intensity distribution. According to this work, delocalization should only contribute to spatial resolution with about $0.5 \mathrm{~nm}$. Our findings using an $18 \mathrm{eV}$ energy-filtered image is in good accordance with this value. We could estimate an overall resolution at $18 \mathrm{eV}$ of about $1 \mathrm{~nm}$. The influence of spherical and chromatic aberration may be neglected except for the $80 \mathrm{eV}$ energy-loss case, where a $20 \mathrm{eV}$ slit width was used. There 
chromatic aberration is estimated to contribute between $5 \%$ and $10 \%$ to the total resolution value.

\section{Conclusions}

In this work, the terms "spatial resolution" and "detection limit" were described with respect to their relation to EFTEM. Length measurements on a $\mathrm{nm}$ or sub-nm length scale depend on spatial resolution, therefore, a knowledge of the value of spatial resolution is required to accurately determine the dimensions of the features analyzed.

The EFTEM investigation of a $\mathrm{Mn} / \mathrm{PdMn}$ multilayer specimen demonstrates the relationship between "real" and "apparent" dimensions and their dependency on spatial resolution. Furthermore, these relationships also allow to give an estimate for the detection limit (in terms of minimum layer thickness in this particular application example).

Using a $\mathrm{SiO}_{2}$ multilayer specimen, the effect of delocalization was shown to have much influence on spatial resolution of low-loss EFTEM images. Even at an energy-loss of $18 \mathrm{eV}$ a spatial resolution of $1 \mathrm{~nm}$ is possible.

\section{Acknowledgements}

Werner Grogger acknowledges support from the Max Kade Foundation for his stay in Berkeley. Work at LBNL/NCEM was supported by the US Department of Energy (contract No. DE-AC0376SF00098). Work at FELMI was financially supported by the Forschungsförderungsfonds für die gewerbliche Wirtschaft, Vienna, Austria. Dr. Jae-Pyoung Ahn and Dr. Gerald Meinhardt, austriamicrosystems, Unterpremstätten, Austria, are gratefully acknowledged for making the $\mathrm{Mn} / \mathrm{PdMn}$ and the $\mathrm{Si}$ oxide test specimens, respectively.

\section{References}

[1] B. Freitag, W. Mader, J. Microsc. 194 (1999) 42.

[2] K.T. Moore, J.M. Howe, D.R. Veblen, Phil. Mag. B 82 (2002) 13.

[3] F. Hofer, P. Warbichler, W. Grogger, Ultramicroscopy 59 (1995) 15.

[4] J. Mayer, D.V. Szabo, M. Rühle, M. Seher, R. Riedel, J. Eur. Ceram. Soc. 15 (1995) 717.

[5] L. Coast-Smith, R. Brydson, P. Tsakiropulos, F. Hofer, W. Grogger, D.V. Dunford, C.M. Ward-Close, Micron 29 (1998) 17.

[6] O.L. Krivanek, M.K. Kundman, K. Kimoto, J. Microsc. 180 (1995) 277.

[7] F. Hofer, P. Warbichler, W. Grogger, O. Lang, Micron 26 (1995) 377.

[8] R. Brydson, F. Hofer, D. Upadhyaya, G. Kothleitner, M. Ward-Close, P. Tsakiropoulos, S. Froess, Micron 27 (1996) 107.

[9] W. Grogger, K.M. Krishnan, R.A. Ristau, T. Thomson, S.D. Harkness, R. Ranjan, Appl. Phys. Lett. 80 (2002) 1165 .

[10] M. Varela, W. Grogger, D. Arias, Z. Sefrioui, C. Leon, C. Ballesteros, K.M. Krishnan, J. Santamaria, Phys. Rev. Lett. 86 (2001) 5156.

[11] H. Kurata, S. Moriguchi, S. Isoda, T. Kobashi, J. Electron Microsc. 45 (1996) 79.

[12] H. Hashimoto, Z.P. Luo, M. Kawasaki, F. Hosokawa, E. Sukedai, Proceedings of the Institute of Physics Electron Microscopy and Analysis Group Conference, 1997, p. 155.

[13] O. Eibl, Ultramicroscopy 69 (1997) 289.

[14] R.F. Egerton, J. Electron Microsc. 48 (1999) 711.

[15] T. Navidi-Kasmai, H. Kohl, Ultramicroscopy 81 (2000) 223.

[16] H. Kohl, A. Berger, Ultramicroscopy 59 (1995) 191.

[17] C. Jeanguillaume, P. Trebbia, C. Colliex, Ultramicroscopy 3 (1987) 237.

[18] F. Hofer, P. Warbichler, W. Grogger, Ultramicroscopy 59 (1995) 15.

[19] H.A. Liebhafsky, H.G. Pfeiffer, E.H. Winslow, P.D. Zemany, X-Rays, Electrons and Analytical Chemistry, Wiley, New York, 1972, p. 349.

[20] B. Schaffer, Dipl. Thesis, Graz University of Technology, 2002.

[21] S.J. Pennycok, Contemp. Phys. 23 (1982) 371.

[22] S.J. Pennycook, D.E. Jesson, A.J. McGibbon, P.D. Nellist, J. Electron Microsc. 45 (1996) 36.

[23] D.A. Muller, J. Silcox, Ultramicroscopy 59 (1995) 195. 